\title{
Pivoting Image-based Profiles Toward Privacy: Inhibiting Malicious Profiling with Adversarial Additions
}

\author{
Zhuoran Liu, Zhengyu Zhao, Martha Larson \\ Radboud University, Netherlands \\ \{z.liu,z.zhao,m.larson\}@cs.ru.nl
}

\begin{abstract}
Users build up profiles online consisting of items that they have shared or interacted with. In this work, we look at profiles that consist of images. We address the issue of privacy-sensitive information being automatically inferred from these user profiles, against users' will and best interest. We introduce the concept of a privacy pivot, which is a strategic change that users can make in their sharing that will inhibit malicious profiling. Importantly, the pivot helps put privacy control into the hands of the users. Further, it does not require users to delete any of the existing images in their profiles, nor does it require a radical change in their sharing intentions, i.e., what they would like to communicate with their profile. Previous work has investigated adversarial images for privacy protection, but has focused on individual images. Here, we move further to study image sets comprising image profiles. We define a conceptual formulation of the challenge of the privacy pivot in the form of an "Anti-Profiling Model". Within this model, we propose a basic pivot solution that uses adversarial additions to effectively inhibit the predictions of profilers using set-based image classification.
\end{abstract}

\section{CCS CONCEPTS}

- Security and privacy $\rightarrow$ Privacy protections; • Computing methodologies $\rightarrow$ Computer vision.

ACM Reference Format:

Zhuoran Liu, Zhengyu Zhao, Martha Larson. 2021. Pivoting Image-based Profiles Toward Privacy: Inhibiting Malicious Profiling with Adversarial Additions. In Proceedings of the 29th ACM Conference on User Modeling, Adaptation and Personalization (UMAP '21), June 21-25, 2021, Utrecht, Netherlands. ACM, New York, NY, USA, 7 pages. https://doi.org/10.1145/3450613.3456832

\section{INTRODUCTION}

Online profiles often contain images, or consist mostly of images, that users have uploaded or liked. Examples include profiles on image-based social media such as Instagram, photo-sharing websites such as Flickr, and dating apps such as Tinder. Users who create such profiles are interested in sharing something about themselves with other people. However, at the same time, they make themselves vulnerable to computer-vision-based profilers, which can automatically infer personal information from images. Although automatic profiling can contribute positively to the value offered by

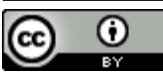

This work is licensed under a Creative Commons Attribution International 4.0 License.

UMAP '21, June 21-25, 2021, Utrecht, Netherlands

(C) 2021 Copyright held by the owner/author(s)

ACM ISBN 978-1-4503-8366-0/21/06.

https://doi.org/10.1145/3450613.3456832 recommender systems, for example, by matching compatible people on a dating site, some profilers are malicious. For example, the site could reinforce the tendency of a person to fall into emotionally abusive relationships. Currently, an increasing amount of research has been carried out on developing profiling technology and recommender systems that protect privacy $[1,15,17]$. However, such research requires users to fully trust the platform provider of the services that they use. There is a lack of research that investigates ways in which control over automatic inference can be placed back into the hands of users.

Our paper addresses this lack by introducing the concept of a privacy pivot for image-based profiles and proposing a basic solution that demonstrates that the predictions of a state-of-theart classifier (used as a profiler) can be inhibited more easily than might generally be assumed. Figure 1 illustrates the privacy pivot concept. A user, Bob, is sharing images online and has accumulated a profile. Bob becomes worried that his personality is being profiled in ways that he does not approve of. Indeed, an automatic profiler predicts that Bob has the personality trait of "Neuroticism" with high confidence (Figure 1 bottom left). At a certain point of time (the pivot point), Bob decides to start uploading photos that will inhibit the profiler. Critically, he does not have the option to delete any existing photos. Although the platform might offer the delete option in the app, there is no guarantee that the existing images are not still in the platform database or somewhere else online. At the pivot point, Bob continues sharing images, but now is doing so strategically to avoid profiling. His pivot is successful because when the automatic profiler makes a prediction on the updated profile (Figure 1 bottom right), it predicts "Neuroticism" with low confidence (i.e., Bob is classified as negative for this personality trait). In this work, we focus on personality, but the idea of pivoting image-based profiles toward privacy is transferable to any personal or privacy-sensitive information.

The main goal of the paper is to provide a framework that will allow computer vision researchers to be able to tackle the challenge of privacy pivot for image-based profiles. The framework, i.e., our "Anti-Profiling Model", is formulated as an adversary model such as used in research on adversarial images. In addition to the AntiProfiling Model, we present a basic pivot based on adversarial addition as a proof of concept. ${ }^{1}$

Currently, adversarial techniques are being used to protect image privacy, but they are limited to inhibiting inference carried out on individual images [21, 30]. Profiler algorithms do not, however, operate on individual images, but rather they are so-called imageset classifiers, which take as input a set of images. In this paper, we

${ }^{1}$ Code for our basic pivot (adversarial addition) and more detailed experimental analysis available at https://github.com/liuzrcc/Image-Set-Profile-Pivot 


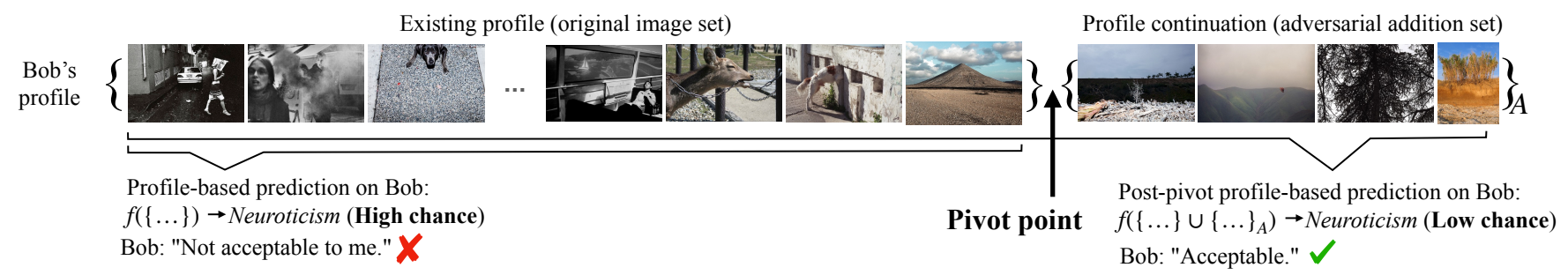

Figure 1: Bob's existing profile leads the automatic profiler to predict Neuroticism (High chance). Bob pivots by continuing his profile using our adversarial addition, achieving a profiling prediction he finds more desirable.

move beyond protecting individual images to lay the ground work for research on protecting entire image-based profiles.

In Section 2, we cover relevant related work. Then, we introduce our "Anti-Profiling Model" in Section 3. Next, in Section 4, we propose a basic adversarial addition pivot and demonstrate its effectiveness at pivoting profiles. We close with a conclusion and outlook in Section 5.

\section{RELATED WORK}

\subsection{Profiling on Social Media}

Machine learning-based approaches have achieved striking success in mining personal information from the accumulated data of user profiles. Here, we mention some key examples. Chaabane et al. [5] show that it is possible to predict private attributes only based on that data that a user reveals, e.g., music that the user has "liked". Further, Kosinski et al. [10] show that easily accessible digital records on the web, e.g., Facebook likes, can be used to predict several private attributes, including religious, personality traits, and gender. Segalin et al. [27] propose a deep learning-based method to predict users' personality traits using only image content. Sertkan et al. [29] explore the possibility of modeling a touristic profile from a selection of a user's pictures based on an aggregated image representation achieved by convolutional neural networks. Han et al. [8] demonstrate that the age and gender of users on Instagram are still identifiable when tags and image content that directly reflects age and gender are excluded. Some private attributes can also be predicted by machine learning through an ego network [13]. Narayanan et al. [18] show that an anonymous online author has other recognizable idiosyncrasies. Although we focus on image profiles here, privacy pivots could be developed for a wide range of cases, including profiles consisting of text or video posts.

\subsection{Protecting User Privacy}

The law is a major source of privacy protection, and research such as [16] has studied the right to be forgotten that is included in Art. 17 of the European General Data Protection Regulation (GDPR). In Europe, users have the right to erase their personal data from the service provider. However, they still must trust that the erasure has taken place and also that their data was not copied elsewhere online. For this reason, research, such as ours, which aims to provide users with direct control over their data, is important, alongside of legal protections. Key examples of related research include: TrackMeNot [19], which adds noise to obfuscate user search engine interaction data, hiding users' actual intention in many "ghost" queries, and, BlurMe [33], which protects user rating profiles by injecting ratings that inhibit inference of user gender. Computer vision researchers have proposed several visual privacy protection methods against person-related recognition [20, 21, 23, 26, 30]. In the area of social images, the Pixel Privacy Task $[12,14]$ promotes image privacy protection methods against malicious scene inference. Privacy-sensitive attributes in images are discussed and conceptualized in [22, 26]. Different from research on adversarial images that prevents inference on individual images, our work focuses on changing the prediction of a image-set classifier by continuing an existing profile, under the condition that images in the existing profile may not be deleted.

\section{ANTI-PROFILING MODEL}

\subsection{General Model}

Privacy and security research makes use of models that provide the specifications of attacks that must be countered. The starting point is a general model consisting of a number of parameters, which provides a framework summarizing the dimensions important for the problem [25]. When proposing a specific solution, researchers specify the settings of the parameters that they are adopting, using motivations based on plausible real-world scenarios. They then formulate their solutions within the model that is defined by the specified settings. The quality of the research is judged both by the motivation for the model settings that are adopted and also by the ability of the solution to prevent attack.

Our point of departure is the observation, mentioned above, that computer vision has potential to address the privacy pivot challenge of image-based profiles if it can move beyond investigating individual images. Specifically, adversarial algorithms could be developed that would allow highly effective pivots. The key piece that is missing before such work can be carried out is the general model that defines how the challenge should be addressed. This model builds on, but is considerably more complex than, single image models. In this paper, we supply this key piece. Technically, this model is an adversary model, since its goal is to inhibit the predictive ability of the image-set classifier. However, we call the model Anti-Profiling Model, since users trying to protect their privacy are not classic adversaries, in the sense that they are not malicious. Our model follows the general structure of adversary models from [25].

The Anti-Profiling Model is specified in Table 1. We describe and motivate each dimension in turn. First, Output dimensions specify properties of the pivoted profile. Output: Prediction describes the 
Table 1: Anti-Profiling Model: A framework specifying the dimensions relevant for pivoting profiles toward privacy.

\begin{tabular}{|c|c|c|}
\hline Dimension & Description & Examples \\
\hline Output: Prediction & $\begin{array}{l}\text { What is the desired behavior of the pivoted profile } \\
\text { with respect to prediction? }\end{array}$ & $\begin{array}{l}\text { Target class should be predicted; } \\
\text { Target class should be avoided. }\end{array}$ \\
\hline Output: Maintenance & $\begin{array}{l}\text { What characteristics of the profile should be main- } \\
\text { tained? }\end{array}$ & $\begin{array}{l}\text { Evidence of pivot appear should be \{imperceptible } \mid \text { nonsuspicious }\} \text { to the human eye; } \\
\text { Pivot should not disrupt what the user would like to share with other people (sharing } \\
\text { intention is maintained). }\end{array}$ \\
\hline Input: Quantity & What is the scope of the existing profile? & $\begin{array}{l}\text { A range specifying the number of images; } \\
\text { The resolution of the images. }\end{array}$ \\
\hline Input: Quality & $\begin{array}{l}\text { What is the structure of the existing profile (in terms } \\
\text { of the nature and distribution of the profile images)? }\end{array}$ & $\begin{array}{l}\text { Few images provide clear evidence for the undesired class; } \\
\text { Most images homogenously provide }\{\text { clear } \mid \text { indistinct }\} \text { evidence for the undesired class. }\end{array}$ \\
\hline $\begin{array}{l}\text { Process: Knowledge } \\
\text { of profiler }\end{array}$ & $\begin{array}{l}\text { How much does the pivoter know about the feature } \\
\text { extractor and the classifier used by the profiler? }\end{array}$ & $\begin{array}{l}\text { Algorithms are known (white box); } \\
\text { Basic facts (gray box); } \\
\text { Nothing (black box). }\end{array}$ \\
\hline $\begin{array}{l}\text { Process: Knowledge } \\
\text { of training data }\end{array}$ & $\begin{array}{l}\text { How much does the pivoter know about the data used } \\
\text { to train the profiler (classifier and feature extractor) }\end{array}$ & $\begin{array}{l}\text { Data is known and available (white box); } \\
\text { Something is known about the data (gray box); } \\
\text { Nothing (black box). }\end{array}$ \\
\hline Process: Resources & What the pivoter capable of doing? & $\begin{array}{l}\text { Computer vision expertise available to the pivoter; } \\
\text { Amount of computational power available. }\end{array}$ \\
\hline $\begin{array}{l}\text { Process: Strength of } \\
\text { pivot }\end{array}$ & $\begin{array}{l}\text { What is the pivoter willing to change in order to } \\
\text { achieve the pivot? }\end{array}$ & $\begin{array}{l}\text { Extent to which the sharing intention is adapted; } \\
\text { Number of images added. }\end{array}$ \\
\hline
\end{tabular}

desired adversarial impact of the pivot, i.e., the nature of misclassification that the pivot should trigger. For example, a successful pivot can be defined as one that classifies a profile into a specific class (i.e., "Healthy") or as one that classifies a profile as any class except a specific class. These choices correspond to the targeted and untargeted cases used in the adversarial image literature [4]. Output: Maintenance describes what should not be changed by the pivot. As in the adversarial image literature, the adversarial transformation should not touch the interpretation of the images, but rather should be either imperceptible [32] or non-suspicious [3]. In the case of a privacy pivot, maintenance applies to the continuation images, since none of the images in the existing profile may be changed. The privacy pivot also allows for a third category: the continuation images can be consistent with specific desiderata, without needing to be specific images. For example, users can pivot by continuing to share images that reflect what they would like to share with others, which we refer to as personal preferences, but which also lean toward the target class. The option to focus on maintaining preferences (sharing intention), rather than maintaining appearance of specific, individual images, does not arise in research on single images.

Second, Input dimensions specify the nature of the content that the pivot must operate upon. In the adversarial image literature, there is little discussion of the full range of characteristics of possible input images. An example of an exception is [36], which discusses the difference between images containing large monochromatic areas and images containing textures. Moving from the single image case to the privacy pivot case, it is no longer possible to gloss over properties of the input. Rather, the existing profile must be specified in terms of ranges that define the number of images it can contain and the resolution of those images (Input: Quantity). It must also be specified in terms of the nature of the images and the distribution of those images (Input: Quality).

The Input: Quality dimension is challenging to specify because it deals with how individual images are interpreted and how the image interpretations come together into a profile interpretation. However, it is important to think carefully about this dimension. A given set of user profiles may be more or less representative in terms of quality of the full diversity of possible profiles. Without attention to quality, vulnerabilities could be easily overlooked. Note that the Input: Quality dimension is not narrowly concerned with the "goodness" of images, but rather encompasses broadly a characterization of what images depict and how they are interpreted.

To make the Input: Quality dimension more concrete, we introduce the notion of profile structure which we consider to arise from the interaction of the nature of the profile images and their distribution. We consider the nature of images to include their semantic content, but also aspects such as style and aesthetics. Taking these aspects into account, we see that there are different ways in which images within the profile can be considered similar to each other. We consider the distribution of the profile to be its level of homogeneity covering all the different possible aspects comprising the nature of images.

We call special attention to the notion of profile clarity, which we define as the degree to which the images in a user profile provide clear evidence that the user falls into the category that the profiler is attempting to infer (i.e., the inference that the pivoter would like to inhibit, such as "Neuroticism" in Fig. 1). This notion of clarity (vs. indistinctness) is important to consider when studying profile privacy, alongside other aspects of images such as semantic content and style, just mentioned. Note that the clarity can be seen as related to individual images, but also to the profile as a whole (since taken together the images provide a complete picture not reflected in the sum of the individual parts). We define clarity in relationship to human judgments of images, but we note that implications of profile clarity for privacy violations will be related to the extent to which the inference ability of a machine learning classifier is related to human-perceived clarity. In Section 4 we explain how we take a specific look at clarity in our experiments.

Third, Process dimensions specify the constraints on the process of generating a pivot. They include classical dimensions related to how much the pivoter knows about the profiler, both in terms of the set-based image classification algorithm being used and in 
terms of the amount of expertise and resources available. Note that it may be the case that a user who would like to pivot their profile has access to a third-party service to help them. In this case, the relevant resources are the resources available to that third party. Process also includes Process: Strength of pivot, which captures what the user is willing to change in order to accomplish the pivot. In the adversarial image literature, the strength of the adversarial attack is related to the bound on the distance that is allowed between the original and the adversarial version of the image, in the case of imperceptible adversarial transformations [7]. If the transformation aims at non-suspiciousness, the strength will be specified by the tolerance of users for certain transformations, and is measured by a user study [2]. In the case of a privacy pivot, we need to also add the number of images that the user is willing to add in the profile continuation and also the degree to which the user might be willing to stray (if at all) from posting images that correspond to their underlying preferences (as defined by the Output: Maintenance).

\subsection{Specific Model}

Next, we describe the settings of the Anti-Profiling Model that we choose to investigate in this paper. We propose a basic pivot based on adversarial addition and experimentally test its effectiveness in inhibiting the prediction performance of a profiler as a user continues to extend their profile.

For Output: prediction, we target a specific class and for Output: maintenance we do not specify that the profile extension contains specific images, but rather that is consistent with the sharing intention (i.e., images are drawn from a preference set of images that the user would have potentially posted). For Input: Quantity, we assume that the existing profile contains 20 images and for Input: Quality we assume the structure of the profile is uncertain (including the nature of the images and the homogeneity of their distribution), but that the data set that we are using is representative of a real-world quality variation.

For Process: Knowledge of classifier, and Process: Knowledge of data we assume no specific knowledge of the classifier other than it is a personality classifier. The pivoter can make, however, a good guess of how the classifier would classify their profile because they are familiar with their own personality or are worried that their profile is reflecting an undesired trait. We study the case in which the pivoter has access to the same image representations as used by the classifier, and also a case in which the pivoter has access to a different set of representations learned on the same data set as used to train the original classifier. Because we use assumptions about the feature representations and the data used to train them, but no information on the classifier, we consider these cases to be gray-box and dark gray-box settings. Note that the data in question is ImageNet data, which is widely available and can be considered currently the default data set for such applications. Assuming ImageNet data is used is therefore not a large leap. Also, in terms of Process: Knowledge of data, we assume that the pivoter has the ability to label data as being "extreme" cases of the target class. This assumption is plausible because personality training data is labeled by human annotators, who are not necessarily themselves experts. Process: Resources we assume that the pivoter is or is being helped by someone with basic knowledge of computer vision. Process: Strength of pivot we assume that the pivoter selects up to 20 images from a set of images that represents their preference. (Note: we chose 20 since it is the maximum reasonable given the resources of our data set.)

\section{EXPERIMENTS}

Our experiments test the ability of adversarial addition to pivot a user profile. The underlying insight motivating adversarial addition is that some of the users' preferred images will be relatively closer to the target value of the personality trait that the user wishes to achieve. The profile will pivot away from the original, undesirable, prediction if the user continues their profile with a selection of these particular images. In this way, users can pursue their sharing intentions while at the same time protecting their privacy.

We test adversarial addition against two profilers, one based on Majority Voting (MV) [27] and one on state-of-the-art Weighted Feature Fusion (WFF) [35]. Note that image-set classifiers that assume that the image set consists of near duplicates or multiple views are not well suited for social media profiling and do not come into consideration for the state of the art. We use two profilers in order to understand how the profiler interacts with the quality (nature and distribution) of the image set that comprises the existing profile. WFF uses a similarity model to calculate the contributions of different images in the profile and then aggregates all images with respect to the calculated weights. The threshold is selected (using the validation set) for each profile length. WFF is able to deal with cases in which a profile contains many low confidence images that should not contribute to the decision.

\subsection{Data and Evaluation}

We perform our experiments on PsychoFlickr data set [28], which is publicly available and widely used for image-based social profiling. PsychoFlickr contains 200 "favored" images from each of 300 Flickr users. Each user's profile is labeled with the Big Five personality traits in psychology: Openness to experience $(\mathrm{O})$, Conscientiousness (C), Extraversion (E), Agreeableness (A), and Neuroticism (N). For each of these five traits, a profile is assigned a binary ground truth label, which is referred to as "attributed trait". The binary label is determined by the magnitude of the scores averaged over all the annotators (10 annotators per profile), which we call the "attributed score". Note that the data set also contains self-assessed traits, but we do not use these here, because we assume the profiler does not have access to self-assessment for training. We also point out that the conclusions of our experiments are independent of our use of the Big Five, i.e., another personality taxonomy could have been chosen. We focus our work, however, on PsychoFlickr, because it was the most suitable publicly available image data set representing personal traits we could find.

We follow the same experimental protocol in [27] to build our classifier (but note our focus is an image-set classifier and not an image classifier). As in [27], our experimental data set is selected to use users for whom the judgments are stronger, i.e., their attributed scores are distributed in first and last quartile. Also, for each personality trait, we select two extreme users (users with highest and lowest attributed score; ties broken using variance) and exclude them from our data to be used for adversarial addition. 
Table 2: Prediction accuracy (\%) averaged on five personality traits (O, C, E, A, N) for user profiles, including original (20 images), ordinary addition, and adversarial addition in both the gray-box and dark gray-box settings. The size (K) of each continuation set is 6, 10, or 20 for both the Majority Voting (MV) and Weighted Feature Fusion (WFF) strategies. Results are reported for total (weak/strong) user types (cf. Section 4.1). The "gray-box" denotes that VGG16 is used as feature extractor when ranking images in the preference set, and "dark gray-box" denotes that AlexNet is used.

\begin{tabular}{|c|c|c|c|c|c|c|c|c|c|c|}
\hline \multirow{2}{*}{$\begin{array}{l}\text { Fusion } \\
\text { Strategy }\end{array}$} & \multirow{2}{*}{ Original } & \multicolumn{3}{|c|}{ Ordinary addition } & \multicolumn{3}{|c|}{ Adversarial addition (gray-box) } & \multicolumn{3}{|c|}{ Adversarial addition (dark gray-box) } \\
\hline & & $\mathrm{K}=6$ & $\mathrm{~K}=10$ & $\mathrm{~K}=20$ & $\mathrm{~K}=6$ & $\mathrm{~K}=10$ & $\mathrm{~K}=20$ & $\mathrm{~K}=6$ & $\mathrm{~K}=10$ & $\mathrm{~K}=20$ \\
\hline MV & $65.3(59.4 / 71.2)$ & $66.5(60.2 / 72.8)$ & $66.7(59.2 / 74.2)$ & $68.7(62.0 / 75.4)$ & $53.0(49.8 / 56.2)$ & $50.4(47.6 / 53.2)$ & $47.9(47.8 / 48.0)$ & $55.2(51.0 / 59.4)$ & $52.9(51.6 / 54.2)$ & $49.2(47.8 / 50.6)$ \\
\hline WFF & $69.5(64.0 / 75.0)$ & $69.8(63.2 / 76.4)$ & $68.6(61.0 / 76.2)$ & $68.7(61.8 / 75.6)$ & $59.6(55.0 / 64.2)$ & $57.2(52.6 / 61.8)$ & $54.5(48.4 / 60.6)$ & $59.9(54.2 / 65.6)$ & $57.0(49.8 / 64.2)$ & $52.4(48.2 / 56.6)$ \\
\hline
\end{tabular}

From the experimental data set, for each of the five personality traits, we sample 20 users for validation and 20 users for the test (in each case 10 positive and 10 negative for that dimension). The remaining data in the experimental data set is designated training data and is used to fine tune an VGG16 [31] image-level classifier trained with ImageNet [6]. This is the best classifer in [27].

In order to evaluate the adversarial addition approach we require test and validation user profiles associated with a preference set (i.e., images that fit the users' sharing intention but were not included in the existing profile). We create "sampled" users for this purpose by sampling from our validation and test users. Recall that there are 200 images from each user. Our sampling process effectively creates multiple users from the original users. A sampled user profile consists of 20 images sampled with replacement. This process gives us 200 "sampled" validation users and 200 "sampled" test users. For each "sampled" user, the images not sampled (i.e., the images remaining from the initial 200) are taken to constitute that user's preference set.

We are interested in whether our basic pivot solution has the same effectiveness across different types of profiles. The way in which the ground truth was created gives us the opportunity to look at differences in the level of clarity at which the evidence for a personality trait is present in profile (related to the dimension Input: Quality of our framework). We assume that this clarity is reflected by the attributed scores assigned by the annotators: "strong" users have higher scores and "weak" users have lower scores. For each of the five personality traits, we separate our 200 "sampled" test users in half based on the size of the attributed score of the user from which they were sampled. In this way, we arrive at a subset of 100 weak "sampled" test users and one of 100 strong "sampled" test users. Each of these subsets consists of 50 users who are positive for the personality trait and 50 who are negative. Note that it would be possible to create subsets of the test data in other ways as well. We chose this approach, since it was straightforward and sufficient to give us some initial insight in the type of variation of pivoteffectiveness we might expect if the nature or distribution of the input profiles changes.

\subsection{Adversarial Addition}

Given one user and a personality trait of this user (which can be positive or negative), we first select the extreme image set (set of images from one of our previously reserved extreme users) associated with the opposite polarity for that personality trait. We calculate the Euclidean centroid of this extreme image set. Then, we select a continuation set from the preference set of the user to be used as an addition to the profile in order to accomplish the pivot. Specifically, for all images in the preference set, we calculate the feature space distance, sort in ascending order, and pick the top $\mathrm{K}$ samples that are nearest to the opposite centroid as the adversarial continuation set. As a comparison, we also construct an ordinary continuation set by randomly sampling $\mathrm{K}$ images from that user's preference set without pivoting.

We test adversarial addition under two settings of the AntiProfiling Model. First, the gray-box setting, where the pivoter has access to the exact feature extractor $z$ that the profiler is using and, second, the dark gray-box setting, where the pivoter has access to a pre-trained ImageNet network, but not to the specific extractor.

We test adversarial addition against two different profiling classifiers, Majority Voting (MV) and Weighted Feature Fusion (WFF). We have balanced test set for our experiments, so we use prediction accuracy of profiler to show the effectiveness of adversarial addition. For all user profiles, we report the average predicted userlevel accuracy. Specifically, for each "sampled" test user with 20 images in profile, we first calculate accuracy on original profile, then we add continuation set consisting of 6,10 , and 20 images to user profile and calculate accuracy again.

\subsection{Experimental Results}

Table 2 presents the average prediction accuracy for original user profiles and profiles extended with continuation sets. "Ordinary addition" shows that the accuracy of the profiler increases as users continue to share images without pivoting. In contrast, our "Adversarial addition" yields decrease of the accuracy, indicating the effectiveness of our proposed pivot. For MV, even in the dark graybox setting the performance can be reduced below $50 \%$ (random chance). We see that WFF is more robust, but the pivot is still highly successful in reducing its accuracy in both the gray-box and dark gray-box settings.

We can also see a clear contrast between the accuracy for the strong and weak users (where weak/strong reflects the relative strength of attributed scores cf. Section 4.1). This contrast shows the importance of our Anti-Profiling Model. If we had ignored the quality dimension, we would not have looked at profiles with different characteristics and would not know that a WFF profiler can be pivoted below $50 \%$ for weak users, but not for strong users (without adding more than the 20 image continuation set, which is the maximum size feasible given our data).

\section{CONCLUSION AND OUTLOOK}

This paper introduces the challenge of privacy pivoting, which has the goal of providing users with control of information that 
automatic profilers extract from their online image-based profiles. We have shown that using a basic approach to pivoting profiles that is based on adversarial addition (under which users continue to share as they wish, but bend their activities toward a target class) is surprisingly effective in inhibiting the prediction performance of state-of-the-art automatic profilers. A key contribution of the paper is the Anti-Profiling Model, which describes the aspects of the problem that are essential to consider when developing privacy pivots. We anticipate that this model will serve to promote work on profile privacy, since it will allow researchers in the computer vision community to understand the problem fully. Here, we have provided only a proof of concept that shows the effectiveness of a basic pivot. Future work will need to explore further to understand the potential and the limitations of our basic pivot with respect to other instantiations of the Anti-Profiling Model and to develop more advanced pivoting approaches.

Future work will also need to investigate other image-set classifiers. We point out that considerations of the nature of the images differentiate the image-set classifiers relevant for this work from the majority of existing image-set classifiers, which assume that images in the set are near duplicates [24, 34, 37] or they assume that there are 1-2 highly-suited images in the set that can be used to make the classification and the others can be discarded $[9,11]$ In the case of social media image profiles, these assumptions may not hold. For example, the different images a user posts to their dating profile are not usually multiple views of the same object. We anticipate that the future will bring the development of new image-set classifiers that are more suited to social media image profiles, and if this happens, research into pivoting image profiles towards privacy will need to keep up.

Moving forward, our Anti-Profiling Model can accommodate other examples of Output: Prediction. Here, we have focused on pivoting to the opposite prediction of a binary classifier. However, in the future, pivots can also be designed to reduce confidence or inhibit regression rather than classification. Also, the case of a profiler attempting to predict a change in a personal attribute, such as a shift in base mood, should also be addressed.

The "Anti-Profiling Model" that we have presented here includes knowledge of the pivoter in the Process dimensions, but does not include knowledge of the profiler. In the future, the model can be extended to also describe the profiler, including, for instance, the profiler's training strategy, types of feature extractor/classifier, and computational resources as well as the profiler's knowledge of the pivoter. Explicitly modeling these dimensions would contribute to the development of stronger pivots. Note that specification of the profiler's knowledge of the pivoter, would help to understand worst-case scenarios and also to support systematic emulation of profilers in order to further enhance the protection strength.

Future research should keep in mind that studying profile pivots needs careful analysis of the real-world conditions in which pivoting is relevant. Specifically, research on adversarial image profiles is not a straightforward extension of research on adversarial images, but rather requires attention to differences that are potentially important. For example, when studying adversarial images, adversarial training is often an obvious next step in order to test the strength of the adversarial effect. However, in the case of adversarial profiles, adversarial training may not be as important since there is not necessarily a clear distinction between changes in user profiles that occur due to pivots and changes that occur due to other reasons. We hope that this paper allows researchers working on adversarial images to understand how to expand from their current focus on image-level classification and extend their methods to set-based image classification.

We close by pointing out that we have formulated Anti-Profiling Model in the context of pivoting image-based profiles. Future work may build on the fact that it is actually equally applicable to other profiles, such as text-based profiles. Further, it can be applied to the problem of creating adversarial videos. Videos consist of frames, meaning that video classification is essentially a set-based image classification problem. Supporting users in protecting their online profiles will require research into all the modalities in which users share information.

\section{REFERENCES}

[1] Ghazaleh Beigi, Ahmadreza Mosallanezhad, Ruocheng Guo, Hamidreza Alvari, Alexander Nou, and Huan Liu. 2020. Privacy-aware recommendation with privateattribute protection using adversarial learning. In ACM International Conference on Web Search and Data Mining. ACM, 34-42. https://doi.org/10.1145/ 3289600.3290962

[2] Anand Bhattad, Min Jin Chong, Kaizhao Liang, Bo Li, and David A Forsyth. 2020. Unrestricted adversarial examples via semantic manipulation. In International Conference on Learning Representations.

[3] Tom B Brown, Nicholas Carlini, Chiyuan Zhang, Catherine Olsson, Paul Christiano, and Ian Goodfellow. 2018. Unrestricted adversarial examples. arXiv preprint arXiv:1809.08352 (2018)

[4] Nicholas Carlini and David Wagner. 2017. Towards evaluating the robustness of neural networks. In IEEE Symposium on Security and Privacy. IEEE, 39-57. https://doi.org/10.1109/SP.2017.49

[5] Abdelberi Chaabane, Gergely Acs, Mohamed Ali Kaafar, et al. 2012. You are what you like! Information leakage through users' interests. In Network and Distributed System Security Symposium. The Internet Society.

[6] Jia Deng, Wei Dong, Richard Socher, Li-Jia Li, Kai Li, and Li Fei-Fei. 2009. ImageNet: A large-scale hierarchical image database. In IEEE Conference on Computer Vision and Pattern Recognition. IEEE, 248-255. https://doi.org/10.1109/ CVPR.2009.5206848

[7] Ian Goodfellow, Jonathon Shlens, and Christian Szegedy. 2015. Explaining and harnessing adversarial examples. In International Conference on Learning Representations.

[8] Kyungsik Han, Yonggeol Jo, Youngseung Jeon, Bogoan Kim, Junho Song, and SangWook Kim. 2018. Photos don't have me, but how do you know me? Analyzing and predicting users on Instagram. In Adjunct Publication of the 26th Conference on User Modeling, Adaptation and Personalization. ACM, 251-256. https://doi.org/ $10.1145 / 3213586.3225232$

[9] Bruno Korbar, Du Tran, and Lorenzo Torresani. 2019. Scsampler: Sampling salient clips from video for efficient action recognition. In IEEE International Conference on Computer Vision. IEEE, 6231-6241. https://doi.org/10.1109/ICCV.2019.00633

[10] Michal Kosinski, David Stillwell, and Thore Graepel. 2013. Private traits and attributes are predictable from digital records of human behavior. Proceedings of the National Academy of Sciences 110, 15 (2013), 5802-5805. https://doi.org/ $10.1073 /$ pnas. 1218772110

[11] Sourabh Kulhare, Shagan Sah, Suhas Pillai, and Raymond Ptucha. 2016. Key frame extraction for salient activity recognition. In International Conference on Pattern Recognition. 835-840. https://doi.org/10.1109/ICPR.2016.7899739

[12] Martha Larson, Zhuoran Liu, Simon Brugman, and Zhengyu Zhao. 2018. Pixel Privacy: Increasing image appeal while blocking automatic inference of sensitive scene information. In Working Notes Proceedings of the MediaEval Workshop. CEUR-WS.org.

[13] Xiaoxue Li, Yanan Cao, Yanmin Shang, Yanbing Liu, Jianlong Tan, and Li Guo. 2017. Inferring user profiles in online social networks based on convolutional neural network. In International Conference on Knowledge Science, Engineering and Management. Springer, 274-286. https://doi.org/10.1007/978-3-319-63558- $3_{2} 3$

[14] Zhuoran Liu, Zhengyu Zhao, and Martha Larson. 2019. Pixel Privacy 2019: Protecting sensitive scene information in images. In Working Notes Proceedings of the MediaEval Workshop. CEUR-WS.org.

[15] Zhifeng Luo and Zhanli Chen. 2014. A privacy preserving group recommender based on cooperative perturbation. In International Conference on Cyber-Enabled Distributed Computing and Knowledge Discovery. IEEE, 106-111. https://doi.org/ 10.1109/CyberC.2014.26 
[16] Alessandro Mantelero. 2013. The EU Proposal for a General Data Protection Regulation and the roots of the 'right to be forgotten'. Computer Law \& Security Review 29, 3 (2013), 229-235. https://doi.org/10.1016/j.clsr.2013.03.010

[17] Frank McSherry and Ilya Mironov. 2009. Differentially private recommender systems: Building privacy into the Netflix prize contenders. In ACM SIGKDD International Conference on Knowledge Discovery and Data Mining. ACM, 627-636. https://doi.org/10.1145/1557019.1557090

[18] Arvind Narayanan, Hristo Paskov, Neil Zhenqiang Gong, John Bethencourt, Emil Stefanov, Eui Chul Richard Shin, and Dawn Song. 2012. On the feasibility of internet-scale author identification. In IEEE Symposium on Security and Privacy. IEEE, 300-314. https://doi.org/10.1109/SP.2012.46

[19] Helen Nissenbaum and Howe Daniel. 2009. TrackMeNot: Resisting surveillance in web search. In Lessons from the Identity Trail: Anonymity, Privacy and Identity in a Networked Society. Oxford: Oxford University Press, 417-436.

[20] Seong Joon Oh, Rodrigo Benenson, Mario Fritz, and Bernt Schiele. 2016. Faceless person recognition: Privacy implications in social media. In European Conference on Computer Vision. Springer, 19-35. https://doi.org/10.1007/978-3-319-46487$9_{2}$

[21] Seong Joon Oh, Mario Fritz, and Bernt Schiele. 2017. Adversarial image perturbation for privacy protection a game theory perspective. In IEEE International Conference on Computer Vision. IEEE, 1491-1500. https://doi.org/10.1109/ ICCV.2017.165

[22] Tribhuvanesh Orekondy, Bernt Schiele, and Mario Fritz. 2017. Towards a visual privacy advisor: Understanding and predicting privacy risks in images. In IEEE International Conference on Computer Vision. IEEE, 3706-3715. https://doi.org/ 10.1109/ICCV.2017.398

[23] Arezoo Rajabi, Rakesh B Bobba, Mike Rosulek, Charles V Wright, and Wu-chi Feng. 2021. On the (Im) Practicality of Adversarial Perturbation for Image Privacy. Proceedings on Privacy Enhancing Technologies 2021, 1 (2021), 85-106. https://doi.org/10.2478/popets-2021-0006

[24] Yongming Rao, Ji Lin, Jiwen Lu, and Jie Zhou. 2017. Learning discriminative aggregation network for video-based face recognition. In IEEE International Conference on Computer Vision. IEEE, 3801-3810. https://doi.org/10.1109/ICCV.2017.408

[25] Chris Salter, O. Sami Saydjari, Bruce Schneier, and Jim Wallner. 1998. Toward a secure system engineering methodology. In Workshop on New Security Paradigms. ACM, 2-10. https://doi.org/10.1145/310889.310900

[26] Hosnieh Sattar, Katharina Krombholz, Gerard Pons-Moll, and Mario Fritz. 2019 Body Shape Privacy in Images: Understanding Privacy and Preventing Automatic Shape Extraction. In European Conference on Computer Vision Workshops. Springer
411-428. https://doi.org/10.1007/978-3-030-68238-531

[27] Cristina Segalin, Dong Seon Cheng, and Marco Cristani. 2017. Social profiling through image understanding: Personality inference using convolutional neural networks. Computer Vision and Image Understanding 156 (2017), 34-50. https: //doi.org/10.1016/j.cviu.2016.10.013

[28] Crisitina Segalin, Alessandro Perina, Marco Cristani, and Alessandro Vinciarelli. 2016. The pictures we like are our image: continuous mapping of favorite pictures into self-assessed and attributed personality traits. IEEE Transactions on Affective Computing 8, 2 (2016), 268-285. https://doi.org/10.1109/TAFFC.2016.2516994

[29] Mete Sertkan, Julia Neidhardt, and Hannes Werthner. 2020. Eliciting touristic profiles: A user study on picture collections. In ACM Conference on User Modeling, Adaptation and Personalization. ACM, 230-238. https://doi.org/10.1145/ 3340631.3394868

[30] Shawn Shan, Emily Wenger, Jiayun Zhang, Huiying Li, Haitao Zheng, and Ben Zhao. 2020. Fawkes: Protecting privacy against unauthorized deep learning models. In USENIX Security Symposium. USENIX Association, 1589-1604.

[31] Karen Simonyan and Andrew Zisserman. 2015. Very deep convolutional networks for large-scale image recognition. In International Conference on Learning Representations.

[32] Christian Szegedy, Wojciech Zaremba, Ilya Sutskever, Joan Bruna, Dumitru Erhan, Ian Goodfellow, and Rob Fergus. 2014. Intriguing properties of neural networks. In International Conference on Learning Representations.

[33] Udi Weinsberg, Smriti Bhagat, Stratis Ioannidis, and Nina Taft. 2012. BlurMe: Inferring and obfuscating user gender based on ratings. In ACM Conference on Recommender Systems. ACM, 195-202. https://doi.org/10.1145/2365952.2365989

[34] Jiaolong Yang, Peiran Ren, Dongqing Zhang, Dong Chen, Fang Wen, Hongdong $\mathrm{Li}$, and Gang Hua. 2017. Neural aggregation network for video face recognition. In IEEE Conference on Computer Vision and Pattern Recognition. IEEE, 5216-5225. https://doi.org/10.1109/CVPR.2017.554

[35] Manyuan Zhang, Guanglu Song, Hang Zhou, and Yu Liu. 2020. Discriminability distillation in group representation learning. In European Conference on Computer Vision. Springer, 1-19. https://doi.org/10.1007/978-3-030-58607-21

[36] Zhengyu Zhao, Zhuoran Liu, and Martha Larson. 2020. Towards Large yet Imperceptible Adversarial Image Perturbations with Perceptual Color Distance. In IEEE Conference on Computer Vision and Pattern Recognition. IEEE, 1036-1045. https://doi.org/10.1109/CVPR42600.2020.00112

[37] Yujie Zhong, Relja Arandjelović, and Andrew Zisserman. 2018. GhostVLAD for set-based face recognition. In Asian Conference on Computer Vision. Springer, 35-50. https://doi.org/10.1007/978-3-030-20890-53 